\title{
Overview of AI in Diagnosis for Schizophrenia, Depression and Anxiety
}

\author{
Shiyu Zuo ${ }^{1, *}$ \\ The University of Manchester, faculty of biology, medicine and health, China, 518000 \\ ${ }^{*}$ Corresponding author. Email: shiyu.zuo@student.manchester.ac.uk
}

\begin{abstract}
The morbidities of mental disorders are becoming higher and higher in the wake of higher and higher urbanization rate. However, the diagnostic accuracies of different mental disorders are not high enough for conducting satisfactory mental health cares. One of the reasons is that, unlike physical illnesses, mental diagnostics relies mainly on symptomatology and rarely on etiology due to the complicated causes of mental disorders. This makes it only possible to use induction rather than deduction during diagnosis. However, the symptoms of the same mental disorders can also vary a lot across patients. In order to do the induction better, it is important and constructive to integrate AI into current psychiatric system. This paper would overview current achievements on diagnosing schizophrenia, depression, and anxiety disorders using AI. In addition, the advantages and shortcomings of utilizing current technologies in real medical scene will also be discussed including diagnostic power, costs and ethical issues.
\end{abstract}

Keywords: artificial intelligence, mental disorders, mental diagnostics, schizophrenia, depression, anxiety

\section{INTRODUCTION}

The rate of mental illness raised along with the increasing rate of industrialization. It has been reported that in 2050, the urbanization rate worldwide will increase from $54 \%$ to $68 \%$ [1]. Although this tendency symbolizes the growing economy, it is a double-edged sword. While urbanizing, people have to face intense changes in lives that are hard to adapt in short time[2]. This leads to increasing stressors, such as higher house rent, larger working stress, and so on. According to previous study [3], the prevalence rate of mental problems was $80.6 \%$ in cities $80.6 \%$ and $48.9 \%$ in villages. The convergent evidences have shown that urbanization is positively correlated to mental illness morbidity [2].

Despite the conclusion is not new, the validity and reliability of current mental diagnostics is still dissatisfactory. The accepted inter-rater reliability should be 0.75 in kappa statistics. However, in DSM-V, the value was lowered to between 0.2 and 0.4 [4]. In particular, diagnosis for Major Depressive Disorder only reached 0.28 [5]. Moreover, the value for MADD was 0.004 [5]. In addition, research found that the specific diagnoses does not predict the outcomes well under many circumsatnces. In fact, receiving diagnosis had very little impact on patients daily lives in many circumstances [6]. Another important statistics is that over $50 \%$ patients were diagnosed with other mental disorders within a year [7]. The high rate of comorbidity allows us to question the underlying theory model and classification methods of psychiatry [8].

One of the potential reasons that cause the embarrassed situation is that, mental diagnostics relies largely on induction and rarely on objective measurements. For example, according to DSM-5[9], patients need to show at least five corresponding symptoms during 2 consecutive weeks to be diagnosed with depression and at least one of the symptoms have to be loss of interest or pleasure or depressed mood. Most of the symptoms are measured through subjective reports, such as Self-Rating Depression Scale [10] and SelfRating Anxiety Scale [11]. Although some physiological measurements are used, they are mainly used to exclude the possibilities of physical illnesses. Therefore, we have reasons to doubt the objectivity of the diagnosis. Another problem is the over reliance on symptomatology and little on etiology. Mental illnesses are not caused by single reason and therefore the certainty is low, i.e. from adverse life experiences to genetic factors [12] or even the combined effects. In fact, the sources can be very different across cases. The diagnosis for physical illnesses is mainly deduction which is a more ideal method. For example, people infected by certain virus 
have pneumonia, Tom is infected, therefore Tom has pneumonia. Most physical illnesses have more steady symptoms so that the premises are more reliable. However, mental illnesses are very different. A classic case is schizophrenia since it contains at least 3 clusters of symptoms which do not correlate with each other. This means that patients can be very different from each other and therefore need different treatments [13]. Hence, it is unrealistic to use deduction mental diagnositics. The diagnosis is more close to induction: Tom has bad mood, this is a symptom of depression, Tom might have depression. Therefore, the more common features we can identify for a given mental disorder, the more accurate the diagnosis would be.

Artificial intelligence is the algorithm that can simulate the problem-solving and decision-making abilities of human intelligence. It is well known for its inducing power and the ability of finding patterns. With the help of AI, it is possible to rely the diagnosis more on objective features. Anhedonia, one of the core symptoms of depression may be originated by the dysregulation of the regulatory afferent circuits of dopamine system [14]. We have reason to believe that more similar features can be found by AI. Hence, it is imperative to explore techinique of AI on mental diagnosis. This paper would overview the achievements that have been made and identify the advantages and shortcomings of applying AI in real medical scene. This paper not only analyzes from the ability aspect, but also discusses ethical issues, costs and so on.

\section{OVERVIEW OF CURRENT AI}

\subsection{Schizophrenia}

It has been suggested that the three important factors in psychiatry-biological, psychological and social factors-can be supported by machine learning [15]. Nowadays, many studies aimed to analyze the data like brain imaging data and EHRs with AI to diagnosis mental disorders and have achieved impressive results [16]. A notable example is EMPaSchiz, an ensemble model for diagnosing schizophrenia which can guarantee $87 \%$ accuracy [17].

Another example is the computer aided diagnosis system built by Chyzhyk, Savio and Graña [18]. This is a system that focus on discriminating schizophrenia by analyzing resting state functional Magnetic Resonance Imaging by applying ensembles of Extreme Learning Machines. The entire process is shown in Figure 1, Overall Working Process of Computer Aided Diagnosis system. Basically, there are three stages in the whole process: 1. Build a 3D correlation map and the voxel site selection mask; 2. Applying the evolutionary wrapper to the whole activity map; 3 . Applying the evolutionary wrapper to the features extracted from the voxel site selection mask and thus connecting the both processes.

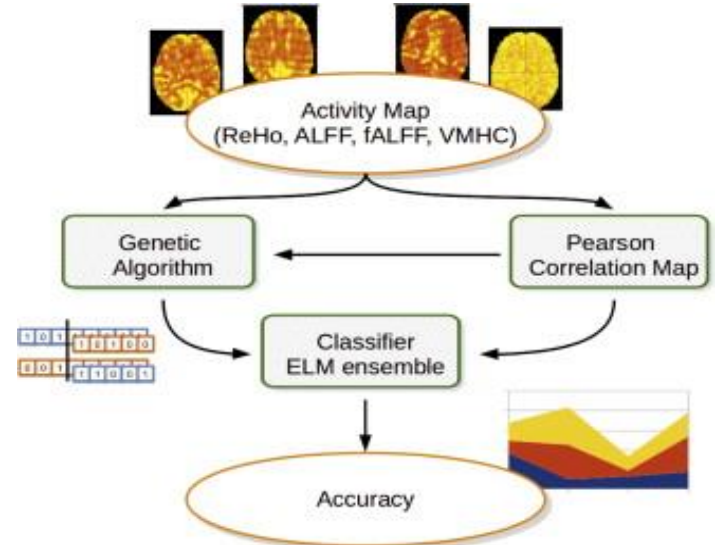

Figure 1 Overall working process of computer aided diagnosis system

\subsection{Depression}

AI diagnosis is not only developed substantially in schizophrenia, but also in depression. A classic example is the deep convolutional neural network set up by Acharya et al. [19]. The neural network contains 5 convolutional layer, 5 pooling layers, and 3 fullyconnected filters with a weighted vector that plays the role of filter and can adjust itself during training. The neural network collects and analyzes the EEG data of both hemispheres of the brains. The results showed that the CNN can yield an accuracy of $93.54 \%$ and $95.49 \%$ respectively by analyzing left and right hemisphere EEG signals.

\subsection{Anxiety}

Similarly, the EEG-based CNN is also used in anxiety prediction. For example, the AnxietyDecoder developed by Wang et al. [20] can distinguish anxious personality and decode the potential biomarkers from the patients. The neural network analyzed the Goal-Conflict-SpecificRhythmicity (GCSR) induced by the task called the Stop Signal Task which can generate anxiety. GCSR is a measure of extent of conflict in brain by using EEG data. By constructing a 3-D CNN with Layer-wise Relevance Propagation, the percentage variance of the Pearson $\mathrm{R}$ between the predictions and the scores on State-Trait Anxiety inventory reached $33 \%$ compared to previous GCSR contrast (7\%).

\section{ADVANTAGES OF USING AI IN MENTAL DIAGNOSIS}

\subsection{Inductive power and data gathering}

The core strength of AI, is its power of gathering and dealing with data. With the help of AI, it is possible to gather and analyze a large amount of medical data for each patient. According to the Biopsychosocial model [21], the diagnosis of mental illnesses should take account of biological, psychological, and social factors. 
However, it is nearly impossible for human psychiatrists to do it perfectly because it would take unimaginable amount of time and energy and the results are very likely to be biased by the subjective views of the psychiatrists towards the qualitative data. On the contrary, machine learning is much better at diagnosing according to Biopsychosocial model [15], which can theoretically pursue the theory better.

\subsection{Time saving and high replicability}

Other preponderances include the time-saving and low-marginal-cost nature of AI. For sophisicated mental illness, only specialist like psychiatrist can identify [22]. However, a psychiatric student needs to be trained at least 13 years to work as a psychiatrist [23]. When taking account of individual differences, the professional abilities vary from psychiatrist to psychiatrist. On the contrary, although it is also time-consuming to train a AI to fit the real patterns of different mental disorders to an acceptable accuracy, it can save a lot of resources and time afterwards while popularizing the technology. The quality of diagnosis in different areas can be ensured.

\subsection{Reconstructing diagnostic system}

Despite the effectiveness of AI has been acknowledged, it is still underestimated. With the help of AI, the underlying model of mental disorders can be reconstructed. Unlike Machine Learning, Deep Learning does not need to analyze on the basis of labelled data, which means that the whole analyzing process does not need to build on any theoretic model. This high level of autonomy allows AI to discriminate patients in a brand new method [17].

\section{SHORTCOMINGS}

\subsection{Unclear Responsibility}

The most obvious problem of using AI, in fact it is a problem in most AI areas, is the ascription of responsibility. Although AI can have very high reliability, the possibility of fault is never eliminated completely. Treating mental patient should be very careful. Woeful results can be produced if the curation is not proper. It has been proposed that the programmers should take all the obligation for the algorithm they produced [24]. Nevertheless, it is a completely different story when we are discussing AI. One of the most classic characteristics of AI is the ability of independent evolution. While it brings AI the ability that exceed human intelligence, it also brings a moral dilemma because even the programmers cannot anticipate the final results. Therefore, it is unsuitable to let them take the responsibility [25]. Moreover, when the AI is installed around the world and used for long time, it is nearly a total certainty that AI will make mistake sooner or later.
As long as this dilemma is not resolved, psychiatrists can only use AI as a counselor which may potentially weaken the efficacy of AI.

\subsection{Lack of sympathy and empathy}

The lack of empathy and sympathy is another big issue that makes it unfitted for real medical scene. While necessary, AI cannot show empathy and sympathy when diagnosing. The common feature of many mental patients are unstable moods which can lead to tragedy. Hence, sympathy and empathy are needed to comfort patients' feelings. Furthermore, empathy is also necessary when detecting the data hidden by the patients. Although discriminating facial expressions can be done fairly accurately by convolution algorithm, it is still hard for them to understand and deal with the moods ingeniously. In addition, sympathy cannot be reduced to compassionate sentences. As long as AI is algorithm and has not reached strong AI level, there is no way for patients to consider them as emotional beings, therefore any comfort would be too weak to take effect. However, there are still many work to do before reaching artificial empathy [26].

\subsection{Increased stigma to patients}

AI can also harm patients by labelling the patients with negative brand. The words used in biomedical model (disorder, symptom) are pathologizing "normal" responses of patients. Being recovered not only means symptoms elimination but also includes stigma dispensation [27]. This is because the brand brought to patients can lead to social anxiety [28]. Moreover, social ostracism can also result in painful consequences [29, 30]. Therefore, it is reasonable to worry about that patients may not emotionally accept to be judged by a machine. This effect can be amplified by the high reliability of AI since it is suggesting that the patients are definitely disordered. It would be putting the cart before the horse if diagnosis itself makes the circumstances even worse.

\subsection{Psychiatrist-AI conflicts}

AI can also bring negative effects to psychiatrists during the curation. Previous study suggested that most of the psychiatrists $(83 \%)$ does not think AI can provide care better than them. Moreover, only $3.8 \%$ of them believed that AI can take over their jobs completely [31]. More surprisingly, half of the psychiatrists do not even deem that AI can bring extensive effect to their jobs. The convergent evidences has shown that the human experts are not ready to cooperate with AI. It can be predicted that, the launch of AI will be opposed by psychiatrists furiously, especially when the results of AI contradict to those of psychiatrists. This would also discourage people to step into this profession because the training time is too long but the future of the career is unsured. 


\section{CONCLUSION}

In this paper, we have reviewed some neural networks that have fairly high accuracy in diagnosing schizophrenia, depression, and anxiety. The advantages and shortcomings of using these networks in real-life scenes are also discussed. From this paper, we can know that although in technological aspects, there is no doubt that we can use the technologies in the near future. However, the technologies are not the barriers that stop them from yielding their highest potentials. The ultimate purpose of diagnosing is to cure. Nonetheless, although it can help, diagnosing better does not mean cure better. Instead, if the shortcomings are not conquered, it is possible that the technologies may even disturb the curation, not only by hurting patients, but also hindering the psychiatrists. We are still far away from replacing psychiatrists completely with AI so if psychiatrists are not ready to work with AI, it is worth to ask to what extent the AI can really help us. Moreover, the unclear accountabilities may result in a crisis of confidence in psychiatrists which make the curation even harder. In conclusion, though AI is powerful, there is still many works to do to bring $\mathrm{AI}$ into real scenes.

\section{ACKNOWLEDGMENT}

First, I am deeply indebted to the teachers in Harbour Education and the professors in my university who provided significant supports on my essay writing and knowledge. Further, I gratefully acknowledge the assistance from Professor Shlomo Ta'asan. It would be impossible for me to complete this paper without his ingenious lectures. I also want to appreciate the help from my friend Szu-han Chen because of his help on my knowledge of AI.

\section{REFERENCES}

[1] UN World Urbanization Prospects. Urban Population at Mid-Year by region, subregion and country, 1950-2050 (thousands), 2018. https://esa.un.org/unpd/wup/Download/.

[2] Srivastava K. Urbanization and mental health. Industrial psychiatry journal, 2009:18(2), 75-76. https://doi.org/10.4103/0972-6748.64028

[3] Reddy M.V, Chandrashekhar C. R. Prevalence of Mental and behavioral disorders in India:a metaanalysis. Indian Journal of Psychiatry, 1998, 40:149-157.

[4] Kraemer, H. C., Kupfer, D. J., Clarke, D. E., Narrow, W. E., \& Regier, D. DSM-5: How reliable is reliable enough? American Journal of Psychiatry, 2012:169(1), 13-15.

[5] Freedman, R., Lewis, D. a, Michels, R., Pine, D. S., Schultz, S. K., Tamminga, C. A, Yager, J. The initial field trials of DSM-5: new blooms and old thorns. The American Journal of Psychiatry, 2013: 170(1), 1-5.

[6] Hanlon FM, Yeo RA, Shaff NA, et al. A symptombased continuum of psychosis explains cognitive and real-world functional deficits better than traditional diagnoses. Schizophr Res. 2019, 208:344-352. doi:10.1016/j.schres.2019.01.024

[7] Kessler, R. C., Chiu, W. T., Demler, O., \& Walters, E. E. Prevalence, Severity, and Comorbidity of 12month DSM-IV disorders in the National Comorbidity Survey Replication. Archives of General Psychiatry, 2005: 62(6), 617-627.

[8] Hyman, S. E. The diagnosis of mental disorders: the problem of reification. Annual Review of Clinical Psychology, 2010: 6, 155-79.

[9] American Psychiatric Association. Diagnostic and statistical manual of mental disorders (5th ed.). Arlington, VA: Author, 2013.

[10] Zung, W. W. A Self-Rating Depression Scale. Arch Gen Psychiatry, 1965: 12, 63-70.

[11] Zung, W. W. A Rating Instrument for Anxiety Disorders. Psychosomatics, 1971:12(6), 371-379.

[12] Mandelli, L., Petrelli, C., \& Serretti, A. The role of specific early trauma in adult depression: A metaanalysis of published literature. Childhood trauma and adult depression. European Psychiatry, 2015: 30(6), 665-680. https://doi.org/10.1016/j.eurpsy.2015.04.007

[13] Liddle, P. F. The symptoms of chronic schizophrenia. A re-examination of the positivenegative dichotomy. The British Journal of Psychiatry, 1987:151(2), 145-151.

[14] Belujon, P., \& Grace, A. A. Dopamine System Dysregulation in Major Depressive Disorders. The international journal of neuropsychopharmacology, 2017: 20(12), 10361046. https://doi.org/10.1093/ijnp/pyx056

[15] Graham, S. A., Lee, E. E., Jeste, D. V., Van Patten, R., Twamley, E. W., Nebeker, C., Yamada, Y., Kim, H. C., \& Depp, C. A. (2020). Artificial intelligence approaches to predicting and detecting cognitive decline in older adults: A conceptual review. Psychiatry research, 284, 112732. https://doi.org/10.1016/j.psychres.2019.112732

[16] Graham, S., Depp, C., Lee, E. E., Nebeker, C., Tu, X., Kim, H. C., \& Jeste, D. V. (2019). Artificial Intelligence for Mental Health and Mental Illnesses: an Overview. Current psychiatry reports, 21(11), 116. https://doi.org/10.1007/s11920-019-1094-0 
[17] Kalmady, S.V., Greiner, R., Agrawal, R. et al. Towards artificial intelligence in mental health by improving schizophrenia prediction with multiple brain parcellation ensemble-learning.npj Schizophr 5, 2

(2019). https://doi.org/10.1038/s41537-018-0070-8

[18] Chyzhyk, D., Savio, A., \& Graña, M. (2015). Computer aided diagnosis of schizophrenia on resting state fmri data by ensembles of elm. Neural Networks, 68, 23-33. https://doi.org/10.1016/j.neunet.2015.04.002

[19] Acharya, U. R., Oh, S. L., Hagiwara, Y., Tan, J. H., Adeli, H., \& Subha, D. P. (2018). Automated EEGbased screening of depression using deep convolutional neural network. Computer Methods and Programs in Biomedicine, 161, 103-113. https://doi.org/10.1016/j.cmpb.2018.04.012

[20] Wang, Y., McCane, B., McNaughton, N., Huang, Z., Shadli, H., \& Neo, P. (2019). AnxietyDecoder: An EEG-based Anxiety Predictor using a 3-D Convolutional Neural Network. 2019 International Joint Conference on Neural Networks (IJCNN). Published. https://doi.org/10.1109/ijenn.2019.8851782

[21] Havelka, M., Despot Lučanin, J. \& Lučanin, D. (2009). Biopsychosocial Model - The Integrated Approach to Health and Disease. Collegium antropologicum, 33 (1), 303-310. Retrieved from https://hrcak.srce.hr/39603

[22] Mind. (2017, October). Mental health problems - an introduction.

https://www.mind.org.uk/informationsupport/types-of-mental-health-problems/mentalhealth-problems-introduction/diagnosis/.

[23] UCAS. (n.d.). Psychiatrist. https://www.ucas.com/ucas/after-gcses/findcareer-ideas/explore-jobs/job-profile/psychiatrist

[24] Bigman, E. Y., Waytz, A., Alterovitz, R., \& Gray, K. (2019). Holding Robots Responsible: The Elements of Machine Morality. Trends in Cognitive Sciences, 23(5), 365-368. https://doi.org/10.1016/j.tics.2019.02.008

[25] Slachta, A. (2019, April 8). The blame game: Who takes responsibility for AI's mistakes? AI in Healthcare. https://www.aiin.healthcare/topics/robotics/whotakes-responsibility-ais-mistakes

[26] Morgan, L. (2018, April 25). AI Challenging: Achieving Artificial Empathy. InformationWeek. https://www.informationweek.com/big-data/ai- machine-learning/ai-challenge-achieving-artificialempathy/a/d-id/1331628

[27] Deegan, P. (1993). Recovering our sense of value after being labeled: Mentally ill. Journal of Psychosocial Nursing and Mental Health Services, 31(4), 7-11.

[28] Read, J., Haslam, N., Sayce, L., \& Davies, E. (2006). Prejudice and schizophrenia: A review of the "mental illness is an illness like any other" approach. Acta Psychiatrica Scandinavica, 114, 303-318.

[29] Williams, K. D. (2002). Ostracism: The power of silence. New York: Guilford Press.

[30] Williams, K. D. (2009). Ostracism: A temporal need-threat model. Advances in Experimental Social Psychology, 41, 279-314.

[31] Doraiswamy, P. M., Blease, C. \& Bodner, K. (2020). Artificial intelligence and the future of psychiatry: Insights from a global physician survey. Artif. Intell. Med. 102, 101753, https://doi.org/10.1016/j.artmed.2019.101 753. 